
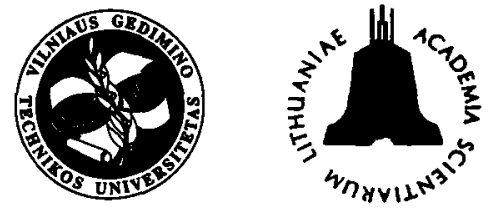

ISSN 1392-3730

JOURNAL OF CIVIL ENGINEERING AND MANAGEMENT

http:/www.vtu.It/english/editions

2003, Vol $I X$, No 2, 83-87

\title{
THE EFFECT OF LOCATION OF SOUND-ABSORBING MATERIALS IN CALCULATING THE REVERBERATION TIME OF THE HALL BY DIFFERENT FORMULAS
}

\author{
Vytautas J. Stauskis \\ Dept of Building Constructions, Vilnius Gediminas Technical University, \\ Traku g.1/26, LT-2001 Vilnius, Lithuania.E-mail: stauskis@ar.vtu.lt
}

Received 21 Feb 2001; accepted 15 Nov 2002

\begin{abstract}
Earlier formulas used for calculating the echoing length in a hall did not make allowance for the location of sound-absorbing materials and the shape of the hall. Fitzroy in 1959 and Neubauer in 1999 tried to take these factors into account. The echoing length calculations were made in a $1018 \mathrm{~m}^{3}$ hall where sound-absorbing materials were placed on the floor and the lateral walls. Formulas produced by 6 authors were used for calculations.

As the echoing length is calculated by Fitzroy's and Neubauer's formulas, with the sound-absorbing materials of different acoustical properties placed on various planes of the hall, large differences in values are obtained compared with the calculations based on Eyring's formula.

When a half of the floor area is covered with a sound-absorbing material, the increase of its absorption ratio results in the increase of the difference in echoing lengths calculated by Fitzroys' and Neubauer's formulas.

When only the lateral walls are covered with sound-absorbing material, the increase of their absorption coefficient from 0,1 to 0,6 results in a $3 \mathrm{~s}$ (or three-fold) increase in the echoing length difference when calculated by Fitzroy's formula and in a $2 \mathrm{~s}$ (or two-fold) increase when calculated by Eyring's formula. Calculation by other formulas gives an insignificant difference of $\sim 0,5 \mathrm{~s}$. As Fitzroy's and Neubauer's formulas are used, the difference becomes more significant: the larger the average hall absorption coefficient, the larger the difference.
\end{abstract}

Keywords: reverberation time, sound absorption materials, absorption coefficient, reflections, energy decay.

\section{Introduction}

Calculation of reverberation times is indispensable for designers of the acoustics for concert, opera theatre, conference and cinema halls as well as for music classes of various sizes. This acoustic indicator is universally accepted and widely used in the acoustical designing practice. Its values depend on the purpose and volume of the hall, acoustical characteristics and arrangement of soundabsorbing materials, and the shape of the hall.

W. Sabine [1] was the first to offer a formula for the calculation of reverberation time (1900). This formula is useful when the average sound absorption coefficient of the hall surfaces is small. In $1930 \mathrm{C}$. F. Eyring [2] improved this formula, which enabled to use it with sufficiently large sound-absorption coefficients, provided that the arrangement of sound-absorbing materials was uniform. If the arrangement is not uniform, the application of Eyring's formula introduces large errors. The formula was further elaborated by A. Millington [3], who assessed the distribution of sound reflections in the air. However, the reverberation time calculated by this formula will be equal to zero if at least one plane is fully sound absorbing. M. C. Gomperts [4] proposed a for- mula that takes into account the early sound reflections. However, these formulas do not allow for the shape of the room and the arrangement of sound-absorbing materials. It was only the formulas worked out by D. Fitzroy [5] and R. O. Neubauer [6-9], and others [10-17] that took the latter factors into consideration. Using these formulas Neubauer calculated and investigated the dependence of reverberation time on hall volume. The calculations do not show the influence of the allocation of materials over reverberation time.

The purpose of this work is to determine the results of reverberation time calculations obtained by the formulas of all the above-mentioned authors when materials with different sound absorption characteristics are allocated on different planes and with different coverage, partial or full.

\section{Formulas for the calculation of reverberation time}

Sabine [1] assumed that the distribution of sound energy in the hall is uniform at any moment, while the surfaces in the hall absorb sound energy uninterruptedly. If the total area of the hall surfaces is $S$ and their absorption coefficient is $\alpha$, then the amount of sound en- 
ergy absorbed per unit of time is

$$
\frac{\alpha S W c}{4},
$$

where $W$ is the density of energy; $c$ is the sound velocity in the air, $\mathrm{m} / \mathrm{s}$.

The energy decay is expressed by the formula

$$
W=\begin{gathered}
4 P \\
\alpha S c
\end{gathered}\left[1-e^{-\frac{\alpha S c}{4 V} t}\right]
$$

The sound field decays exponentially, which is described by the formula

$$
f_{S}(t)=\exp \left[-\left(\frac{c \alpha S}{4 V} t\right)\right] .
$$

This means that both growth and decay of the sound field is exponential, with the sound field being fully diffusive. The larger the $\alpha$ value, the slower the field growth rate. The reverberation time is calculated using Sabine's formula

$$
T=\begin{aligned}
& 0,163 \mathrm{~V} \\
& \sum S_{i} \alpha_{i}
\end{aligned} .
$$

The formula has been derived on an assumption that the energ absorption is quite slow, ie the absorption coefficient a is sufficiently small for a uniform distribution of energy. The pre-condition is that the sound field is diffusive and the hall shape is regular.

When the absorption coefficient $a$ is sufficiently large $(\alpha>0,2)$ this formula gives errors. Therefore, Eyring [2] concluded that sound absorption should be treated as a process with interruptions occurring every time when the sound wave is reflected from the surface. However, in this case it is also recognised that the distribution of energy in the room is uniform and the sound waves travel out in all directions, ie the sound field must be fully diffusive and its decay must be exponential and expressed by the formula:

$$
T=\begin{gathered}
0,163 V \\
-\sum S \ln (1-\alpha)
\end{gathered} .
$$

This formula differs from Sabine's formula in that the absorption coefficient $\alpha$ is replaced by the function $(1-\alpha)$. Where the values of a are low, both these formulas coincide.

Eyring's formula was derived on an assumption that the positioning of sound-absorbing materials is uniform and the energy decay is exponential according to the formula:

$$
f_{E}(t)=\exp \left[-\left(\begin{array}{l}
c S \\
4 V
\end{array} \ln (1-\alpha)^{-1}\right)\right] .
$$

If the arrangement of sound-absorbing materials is not uniform, the above formula may introduce significant errors.
Millington [3] tried to assess a non-uniform arrangement of sound-absorbing materials by adopting $n$ number of reflections with the sound absorption coefficients $\alpha_{n}$. Then the decrease in energy is:

$$
E_{a}=E_{0}\left(1-\alpha_{1}\right) \frac{c S_{1}}{4 V} t\left(1-a_{2}\right) \frac{c S_{2}}{4 V} t
$$

The decay of the sound field must be exponential as expressed by the following equation:

$$
f_{M}(t)=\exp \left[-\left(\frac{c}{4 V} \sum_{i} S_{i} \ln \left(1-\alpha_{i}\right)^{-1}\right)\right] .
$$

The reverberation time is calculated as follows:

$$
T_{M}=\begin{gathered}
0,163 V \\
-\sum S_{i} \ln \left(1-\alpha_{i}\right)
\end{gathered} .
$$

Gompert [4] proposed the following formula for the calculation of reverberation time:

$$
T_{G}=-{ }_{c S}^{4 V}\left(\begin{array}{c}
\sigma^{2} \ln (1-\alpha) \\
2
\end{array} \quad \begin{array}{c}
\ln 10^{4} \\
\ln (1-\alpha)
\end{array}\right) .
$$

In this case the sound field decay must also be exponential as expressed by this equation:

$$
f_{G}(t)=\exp \left(-{ }_{4 V}^{c S} \alpha_{v} t\right) .
$$

Fitzroy [5] offered a formula for calculating the reverberation time that allows for the shape of the room and the positioning of sound-absorbing materials:

$$
\begin{gathered}
T=\left(\begin{array}{c}
X \\
S
\end{array}\right)\left[\begin{array}{c}
0,163 V \\
-S \ln \left(1-\alpha_{x}\right)
\end{array}\right]+\left(\begin{array}{c}
Y \\
S
\end{array}\left[\begin{array}{c}
0,163 V \\
-S \ln \left(1-\alpha_{y}\right)
\end{array}\right]+\right. \\
\left(\begin{array}{l}
Z \\
S
\end{array}\left[\begin{array}{c}
0,163 V \\
-S \ln \left(1-\alpha_{z}\right)
\end{array}\right]\right.
\end{gathered}
$$

where $X, Y, Z$ are the areas of planes parallel to $X, Y$ and $Z$ axes respectively, $\mathrm{m}^{2} ; S$ is the total area of the planes, $\mathrm{m}^{2} ; V$ is the volume, $\mathrm{m}^{3} ; \alpha_{x} \alpha_{y} \alpha_{z}$ are the average sound absorption coefficients of the planes parallel to $X, Y$ and $Z$ axes respectively.

Then the function of sound absorption can be determined from:

$$
\ln (1-\alpha)=\frac{S}{\frac{X}{-\ln \left(1-\alpha_{x}\right)}+\frac{Y}{-\ln \left(1-\alpha_{y}\right)}+\frac{Z}{-\ln \left(1-\alpha_{z}\right)}}
$$

Neubauer [6-9] proposes such equation for the calculation of reverberation time:

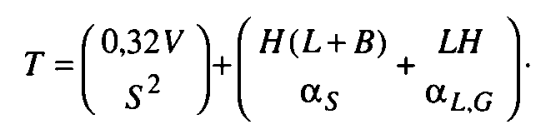

The average sound absorption ratio for the walls is calculated by the formula

$$
\alpha_{S_{(v)}}=-\ln \left(1-\alpha_{(v)}\right)+\left[\begin{array}{c}
\rho_{S}\left(\rho_{S}-\rho_{v}\right) S_{S}^{2} \\
\left(\rho_{v} S\right)^{2}
\end{array}\right] .
$$


The average sound absorption ratio for ceilings and floors is calculated by the formula

$$
\alpha_{L, G_{(v)}}=-\ln \left(1-\alpha_{(v)}\right)+\left[\begin{array}{c}
\rho_{L, G}\left(\rho_{L, G}-\rho_{v}\right) S_{L, G}^{2} \\
\left(\rho_{v} S\right)^{2}
\end{array}\right],
$$

where $\alpha_{v}$ is the arithmetic average of sound absorption of all the planes; $p=(1-\alpha)$ is the sound reflection coefficient; $S_{S}=2 L^{*} H+2 H^{*} B$ is the total area of the walls, $\mathrm{m}^{2} ; S_{L, G}=2 L \times B$ is the total area of the floor and the ceiling, $\left.\mathrm{m}^{2} ; S=\left[H(L+B)+l^{*} b\right)\right]$ is the total area of all the surfaces, $\mathrm{m}^{2}$.

Using this formula Neubauer calculated and investigated the dependence of reverberation time on hall volume. The calculations do not show the influence of the allocation of materials over the reverberation time.

\section{Results of calculations}

A hall $13,6 \mathrm{~m}$ long, $10,7 \mathrm{~m}$ wide and $7 \mathrm{~m}$ high was selected for calculations. The hall volume is $1018 \mathrm{~m}^{3}$. The formulas proposed by all the six authors mentioned above were used for the calculations. In today's acoustical designing practice, Eyring's formula is most widely applied, and we will focus on this formula as well as the ones proposed by Fitzroy and Neubauer. According to these authors, these equations allow for different arrangement of sound-absorbing materials.

For the purpose of calculations, sound absorbing materials were placed on a lateral wall, while other planes were without such materials. Similarly, the back wall or the front wall, or $50 \%$ or $100 \%$ of the ceiling/floor area were covered with a sound-absorbing material. An assumption was made that differences in the results of calculations made by means of all the six formulas would enable to determine whether Fitzroy's and Neubauer's formulas provide a correct allowance for different positioning of sound-absorbing materials and the change in the absorption coefficients.

Thus reverberation time is influenced by positioning materials with different sound-absorption coefficients on various surfaces of the hall. The larger the soundabsorption coefficient of such materials, the larger the difference in the reverberation time values obtained by Eyring's, Fitzroy's and Neubauer's formulas.

Fig 1 depicts the reverberation time dependence upon the change in the hall average sound absorption coefficient, with the reverberation time calculated by different formulas and a half of the floor area covered by different sound-absorbing materials.

When sound absorption coefficient of a half of the floor area is 0,1 , reverberation time obtained from Eyring's formula is 5,33. When calculated by Millington's formula, it is shorter by $0,67 \mathrm{~s}$ than the Eyring's result, and by Fitzroy's and Neubauer's formulas - longer by $0,21 \mathrm{~s}$ and $0,34 \mathrm{~s}$ respectively. The difference between the calculated values increases along with the increase in the absorption coefficient. When the floor absorption

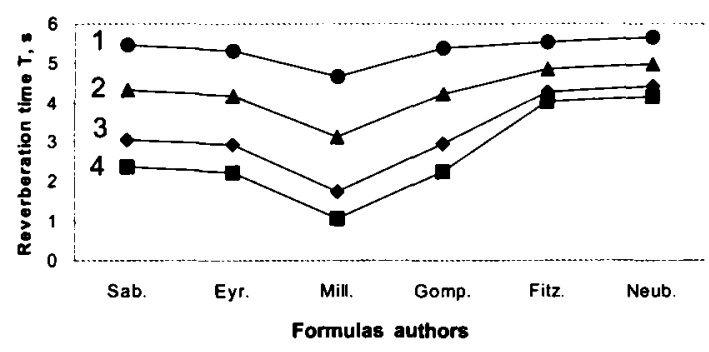

Fig 1. Dependence of reverberation time on the variations of the sound-absorption coefficient and the formulas used for calculations. $50 \%$ of the floor area covered with sound-absorbing material; $50 \%$ of the ceiling area; one lateral wall; the back wall. 1, 2, 3 and 4 - soundabsorption coefficients of respective planes 0,$1 ; 0,2 ; 0,4$ and 0,6

coefficient is 0,6 , the differences increase to 1,81 and $1,94 \mathrm{~s}$ respectively. Thus the larger the sound absorption coefficient, the larger values are obtained by the last two formulas.

Fig 2 shows differences in reverberation times depending on varying average absorption coefficients.

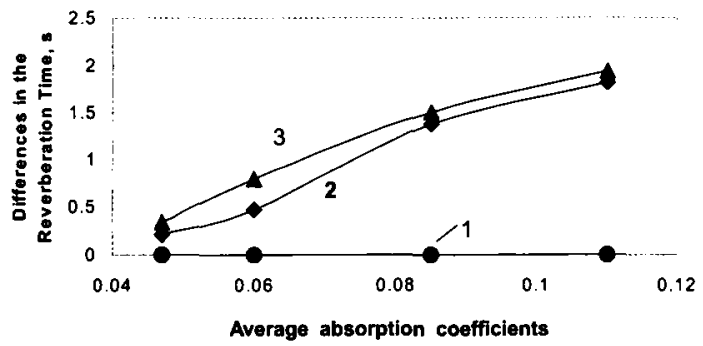

Fig 2. Differences in the reverberation time depending on the change in the average sound absorption coefficient as calculated by different formulas 1 - Eyring's formula; 2 Fitzroy's formula; 3 - Neubauer's formula

One sees that, at the average hall's absorption coefficient of 0,047, Fitzroy's and Neubauer's formulas provide values that are larger than those obtained by Eyring's formula by $0,2-03 \mathrm{~s}$. When the average absorption coefficient increases to 0,11 , the values of reverberation times double, ie are larger by about $2 \mathrm{~s}$.

Fig 3 depicts results obtained when a half of the wall, floor and ceiling surfaces are covered with materials whose $\alpha=0,1$.

The lowest reverberation time values are obtained by Millington's formula, with a half of ceiling and floor planes covered with sound absorbing materials. When calculated by Fitzroy's and Neubauer's formulas, the values are by about 0,3 larger than those calculated by Eyring's formula.

Fig 4 shows changes in reverberation values when materials with different absorption coefficients are placed on both lateral walls.

In this case, when the absorption coefficient increases from 0,1 to $0,6 \mathrm{~s}$, small difference between the 


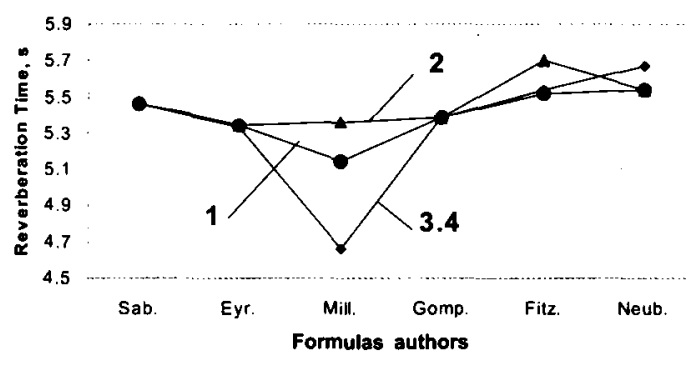

Fig 3. The dependence of the reverberation time on different options of planes covering with sound-absorbing materials as calculated by different formulas. The hall's average sound absorption coefficient equal 0,$047 ; 0,5$ of the area of the hall's lateral, back and front walls, ceiling and floor $\alpha=0,1$; remaining surfaces $\alpha=0,04$. 1 - lateral walls; 2 - front and back walls; 3, 4 - ceiling and floor

reverberation time values is obtained by Sabine's, Eyring's, Millington's and Gompert's formulas. However, with Fitzroy's and Neuebauer's formulas the difference increases along with the increase in the hall's average absorption coefficient. When the absorption coefficient of the lateral walls is equal to 0,1 , the difference amounts to just about $0,5 \mathrm{~s}$, when it equals 0,6 - the difference is about $3 \mathrm{~s}$ (threefold) when calculated from Fitzroy's formula and about $2 \mathrm{~s}$ (twofold) when calculated by Neubauer's formula.

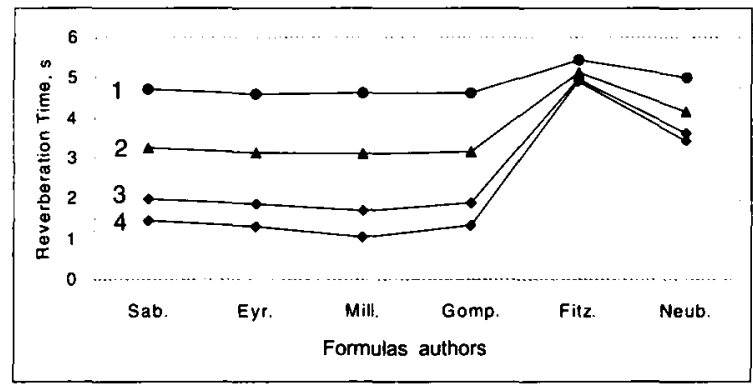

Fig 4. The dependence of the reverberation time on the change in the sound absorption coefficients as calculated by different formulas. Sound-absorbing materials placed throughout lateral walls. The absorption coefficient for all the other planes is equal to $0,04.1-\alpha_{\mathrm{gr}}=0,1 ; 2-\alpha_{\mathrm{gr}}=0,2$; $3-\alpha_{\mathrm{gr}}=0,4 ; 4-\alpha_{\mathrm{gr}}=0,6$. The hall's average sound absorption coefficients are $0,055,0,08,0,13$ and 0,18 respectively

Fig 5 shows the dependence of the differences in reverberation times upon the change in the hall's average sound absorption coefficient.

Compared with the results obtained by Eyring's formula, the difference in the reverberation times increases along with the increase in the hall's average coefficient. The difference is larger when calculated by Fitzroy's formula and smaller - by Neubauer's formula.

The change in reverberation time when different planes are covered with absorbing materials is shown in Fig 6.

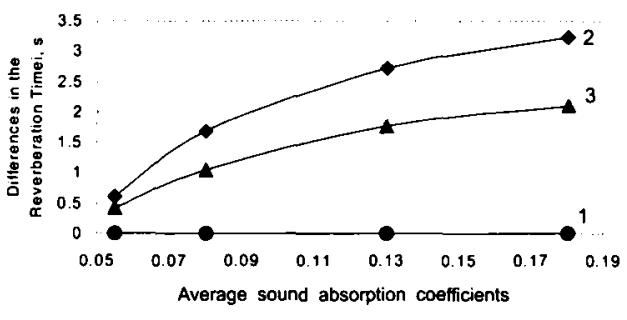

Fig 5. Differences in the reverberation time depending on the change in the average sound absorption coefficient as calculated by different formulas. 1 - Eyring's formula; 2 Fitzroy's formula; 3 - Neubauer's formula

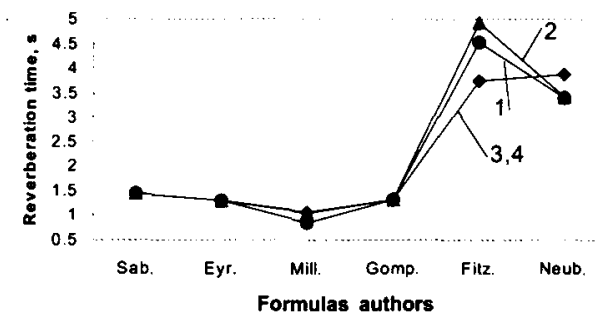

Fig 6. The dependence of the reverberation time on different options of planes' covering with sound-absorbing materials as calculated by different formulas. The hall's average sound absorption coefficient is equal to 0,047 ; both lateral walls, front and back walls, ceiling and floor of the hall covered with materials where $\alpha=0,6$, other surfaces $\alpha=0,04$. 1 - lateral walls; 2 - front and back walls; 3, 4 - ceiling and floor

When the absorption coefficient of two lateral walls amounts to $0,6 \mathrm{~s}$ and that of other planes to $0,04 \mathrm{~s}$, a large (about threefold) difference in reverberation times is obtained by Fitzroy's formula, while an approximately twofold difference - by Neubauer's formula. The allocation of sound-absorbing materials on the planes has great influence on the reverberation time when calculated by the above-mentioned formulas and comparing the results with the Eyring's formula.

\section{Conclusions}

1) When the floors and lateral walls are covered with materials by small absorption coefficients, small difference in reverberation times is obtained by all the formulas. As the coefficients increase, the difference between the values calculated by Eyring's, Fitzroy's and Neubauer's formulas increases.

2) Where reverberation time is calculated by Fitzroy's and Neubauer's formulas, with the materials of different acoustical properties allocated on different planes of the wall, a large difference in values is obtained compared with Eyring's formula.

3) Where a half of the floor plane is covered by sound absorbing material, the increase in its sound absorption coefficient increases the difference in reverbera- 
tion times calculated from Fitzroy's and Neubauer's formulas.

4) When only lateral walls are covered with sound absorbing materials, the increase in their absorption coefficient from 0,1 to 0,6 results in a $3 \mathrm{~s}$ difference (threefold increase) calculated by Fitzroy's formula and a $2 \mathrm{~s}$ difference (twofold increase) calculated by Neubauer's formula. If other formulas are used, the difference increases along with the increase in the average hall absorption coefficient.

\section{References}

1. Sabine, W. Amer. arch. and building news. New York, 1900. 481 p.

2. Eyring, C. F. Reverberation time in "dead" rooms. $J$. Acoust. Soc. Am., 1930, 1, p. 217-226.

3. Millington, A. A modified formula for reverberation. $J$. Acoust. Soc. Am., 1932, p. 69-82.

4. Gomperts, M. C. Do the classical reverberation formulae still have a right for existence? Acustica, 1965 (66, 16(5), p. 255-268.

5. Fitzroy, D. Reverberation formula which seems to be more accurate with non-unform distribution of absorption. $J$. Acoust. Soc. Am., 1959, 31, p. 893-902 .

6. Neubauer, R. O. Prediction of reverberation time in rectangular rooms with non-uniformly distributed absorption using a new formula. In: EAA Symposium on Architectural Acoustics, Il Iberoamerican Congress on Acoustics. 2000 October 16-20. Madrid, p. 49-56.

7. Neubauer, R. O. Prediction of reverberation time in rectangular rooms with modified Fitzroy equation. 8th international symposium on sound engineering and mastering. 1999 Sept 09-11. Gdansk, p. 115-122.
8. Neubauer, R. O. Classroom acoustics - do existing reverberation time formulae provide reliable values. In: 17-th International Congress on Acoustics. Roma, Sept 2-7, 2001, Italy. Vol 25 N1-3/2001, p. 360.

9. King, J.; Neubauer, R. O. Predicting reverberation time: comparison between analytic formulae and computer simulation. In: 17-th International Congress on Acoustics. Roma, Sept 2-7, 2001, Italy, Vol 25, N1-3/2001, p. 361.

10. Mulder, C. Variable acoustics using multiple channel amplification of reverberation time. In: 17-th International Congress on Acoustics, Roma, Sept 2-7, 2001, Italy, Vol 25, N1-3/2001, p. 360.

11. Lim, M. K. at Lehmann R. Revue critique des theories de la reverberation. Revue on Acoustics, 1982, Vol 4, p. 293302.

12. Antonio, J.; Godinho, L.; Tadeu, A. Reverberation times obtained using a numerical model versus those given by simplified formulas and measurements. Acta Acustica, Vol 88920000 , p. 252-261.

13. Hodgson, M. Evidence of difuse surface reflections in room. J. Acoust. Soc. Am., 89, 1991, p. 765-771.

14. Hodgson, M. On the prediction of sound fields in large empty rooms. J. Acoust. Soc. Am., 84, 1988, p. 253-261.

15. Kuttruf, H. Room acoustics. Applied science, London, 1976.

16. Dance, S. M.; Shield, B. M. Modelling of sound fields in enclosed spaces with absorbent room surfaces, Part II, absorptive panels. Applied Acoustics, 61, 2000, p. 373-384.

17. Beranek, L.; Hidaka, T. Sound absorption in concert halls by seats, occupied and unocuppied, and by the hall's interior surfaces. J. Acoust. Soc. Am., 104, 1998, p. 30693117. 